\title{
Damping of hydromagnetic waves by bulk viscosity
}

\author{
M. H. Ibáñez S. \\ Centro de Física Fundamental, Facultad de Ciencias, Universidad de los Andes, La Hechicera, \\ Mérida, Venezuela \\ email: mhibanez@yahoo.com
}

\begin{abstract}
It was shown in a previous paper (Ibáñez 2004) that for certain kind of plasmas the coefficient of second (bulk) viscosity can be orders of magnitude larger than the coefficients corresponding to the dynamical viscosity and to the thermometric conductivity. In the present paper, the damping effects of the second viscosity on the hydromagnetic waves propagation are analyzed. In particular, we study the effect of the second viscosity on the magnetoacustic waves propagating in a photoionized gas of arbitrary metallicity $Z$ and mean energy $E$ of the ionizing photons when an initial steady magnetic field $\mathbf{H}$ is present.
\end{abstract}

Keywords. waves, plsmas, MHD

\section{Introduction.}

Propagation of hydromagnetic waves is an important topic in many different contexts, in particular in astrophysical plasmas, for instance in the solar atmospher (Roberts 2000, Moortel \& Hood 2003, Carbonell et al. 2004, Kumar et al. 2006, Kumar\& Kumar 2006), in the different regions (say, cold warm, hot regions and coronal gas)(Hannebelle \& Passot 2006), as well as in clouds of high velocity in our galaxy where a mean magnetic field of about $2-6 \mu G$ (Spitzer 1978, Parker 1979, Heiles \& Troland 2005) exists. Magnetohydrodynamic (MHD) waves can generate turbulent motions as can also become sources of heat when they are dissipated in the plasma by the different irreversible processes as heat conduction, dynamical and bulk viscosities. This last dissipative process is in general neglected and most of the works on this subject take into account only the dynamical viscosity and thermal diffusivity none the less the bulk viscosity is the dominant dissipative process in several kind of plasmas, in particular, in a recombining gas, as it was shown in a previous work (Ibáñez 2004).

The damping effect on the acoustic waves by bulk viscosity was worked out in a previous paper (Ibáñez 2004). At the present work, the previous papers (Ibáñez \& Escalona 1993 and Ibáñez 2004) are generalized, to accounting the damping effects of the second viscosity on the MHD waves, (effects generally neglected in works on damping of MHD waves in astrophysical as well as laboratory classical plasmas). For context, a photoionized hydrogen gas will be analyzed.

\section{Basic Equations}

It has been well established that hydromagnetic waves propagate though plasmas where magnetic fields are present. In fact, consider a static fluid in a steady state defined by a magnetic field $\mathbf{H}$, a pressure $p$ and a density $\rho$, small adiabatic fluctuations of density $\rho^{\prime}$, pressure $p^{\prime}$, and velocity $\mathbf{v}$, where a magnetic field $\mathbf{h}$ is assumed to be superposed. Then the linearized MHD equations are simplified to 


$$
\begin{gathered}
\operatorname{div} \mathbf{v}=0, \quad \frac{\partial \mathbf{h}}{\partial t}=\operatorname{curl}(\mathbf{v} \times \mathbf{h}), \\
\frac{\partial \rho^{\prime}}{\partial t}+\rho \nabla \cdot \mathbf{v}=0, \\
\frac{\partial \mathbf{v}}{\partial t}=-\frac{u_{0}^{2}}{\rho} \nabla \rho^{\prime}-\frac{1}{4 \pi \rho}(\mathbf{H} \times \operatorname{curl} \mathbf{h}),
\end{gathered}
$$

where the magnetic field is assumed to be frozen in the plasma, i.e. the conductivity $\sigma \rightarrow \infty$ (Landau \& Lifshitz 1966, Braginskii 1965). If, additionally, the fluctuations are assumed to be of the form of plane waves $\sim \exp [i(\mathbf{k} \cdot \mathbf{r}-\omega t)]$ where the wave number $\mathbf{k}$ is taken along the $x$-axis (without losing of generality) and the magnetic field $\mathbf{H}$ lays on the plane $x, y$, the equations $(2.1)$ are further simplified to

$$
\begin{gathered}
u h_{z}=-v_{x} H_{x}, \quad u v_{z}=-\frac{H_{x} h_{z}}{4 \pi \rho}, \\
u h_{y}=v_{x} H_{y}-v_{y} H_{x}, \quad u v_{y}=-\frac{H_{x} h_{y}}{4 \pi \rho}, \\
v_{x}\left(u-\frac{u_{0}^{2}}{u}\right)=\frac{H_{y} h_{y}}{4 \pi \rho},
\end{gathered}
$$

where $u_{0}^{2}=(\partial p / \partial \rho)_{s}$ is the isentropic sound velocity and $u=\omega / k$ is the phase velocity.

Equations (2.2) define Alfvén's waves propagating with velocity

$$
u=a_{x}=\frac{H_{x}}{\sqrt{4 \pi \rho}}=a \cos (\theta),
$$

where $a$ is the Alfvén velocity and $\theta$ is the angle between $\mathbf{H}$ and $\mathbf{k}$.

On the other hand, the set of Eqs. (2.3) and (2.4) define the magnetosonic waves for which the compatibility condition gives

$$
u_{f, s}^{2}=\frac{1}{2}\left\{a^{2}+u_{0}^{2} \pm\left[\left(a^{2}+u_{0}^{2}\right)^{2}-4 a^{2} u_{0}^{2} \cos ^{2}(\theta)\right]^{1 / 2}\right\},
$$

where the plus and minus signs in Eq. (2.6) correspond to fast and slow magnetosonic waves, respectively (Landau \& Lifshitz 1966). The two groups of waves above propagate independently of each other.

\section{Damping of Magnetosonic Waves by Bulk Viscosity.}

It can be easily realized that if one takes into account thermal diffusion of heat as well as the dynamical and bulk viscosities, they only affect the magnetosonic modes. Then, to evaluate the associated damping effect one can generalize the analysis carried out in the previous Section by introducing the corresponding viscosity terms in the equation of motion. The corresponding terms in the energy equation are second order terms, and therefore they can be neglected. However, due to the fact that the bulk viscosity coefficient exhibits dispersion, it is simpler to apply the Landau and Lifshitz method (Landau \& Lifshitz 1987) that has also been applied in the analysis of the damping of sound waves by bulk viscosity (Ibáñez 2004). That is, instead of the equation of state, 
one considers a motion in which viscosity is neglected but the pressure $p^{\prime}$ is given by the formulae

$$
p^{\prime}=\frac{\tau \rho}{1-i \omega \tau}\left(c_{0}^{2}-c_{\infty}^{2}\right) \nabla \cdot \mathbf{v}
$$

where $\tau$ is the relaxation time

$$
\tau=[\partial X(\rho, T, \xi) / \partial \xi]^{-1}
$$

$X(\rho, T, \xi)$ is the net chemical rate and $\xi$ is the chemical parameter denoting the net advance of the reactions. The sound velocities $c_{\infty}^{2}$ and $c_{0}^{2}$ are defined by

$$
c_{\infty}^{2}=\left(\frac{\partial p}{\partial \rho}\right)_{\xi}, \quad c_{0}^{2}=\left(\frac{\partial p}{\partial \rho}\right)_{e q}=\left(\frac{\partial p}{\partial \rho}\right)_{\xi}+\xi_{0}^{\prime}\left(\frac{\partial p}{\partial \xi}\right)_{\rho},
$$

where $\xi_{0}$ is the value of the chemical parameter at chemical equilibrium and $\xi_{0}^{\prime}=$ $\left(\partial \xi_{0} / \partial \rho\right)$, also at equilibrium.

Formally, $k=\omega / u_{0}$ remains applicable but now the expression for $u_{0}^{2}$ involved in Eq. (2.6)] must be changed to $c^{2}$, where

$$
c^{2}=\left(\frac{\partial p}{\partial \rho}\right)=\frac{c_{0}^{2}-i \omega \tau c_{\infty}^{2}}{1-i \omega \tau},
$$

The quantity $c$, however, no longer denotes the velocity of sound being complex (Landau \& Lifshitz 1987).

If the Alfvén velocity $a$, as well as $c$, are normalized to the sound velocity $c_{0}$ and the frequency $\omega$ is normalized to $1 / \tau$ such that $a^{2}=H^{2} /\left(4 \pi \rho c_{0}^{2}\right)$, and

$$
c^{2}=\frac{1+\beta^{2} \omega^{2}}{1+\omega^{2}}+i \frac{\left(\omega-\beta^{2} \omega\right)}{1+\omega^{2}},
$$

where $\beta^{2}=c_{\infty}^{2} / c_{0}^{2}$, then Eq. (2.6) still holds, but with $u_{f, s}$ being a complex quantity also normalized to $c_{0}$.

On the other hand, for magnetosonic waves the relation $k=\omega / u_{f, s}$ remains valid. Therefore, for the fast and slow magnetosonic waves $k_{f}=k_{f 1}+i k_{f 2}$, and $k_{s}=k_{s 1}+i k_{s 2}$, respectively, where $k_{j m}(j=f, s$ and $m=1,2)$ are real quantities. All the above wave numbers are assumed to be normalized to the wave number $k_{\tau}=1 / \tau c_{0}$; consequently, the phase velocity also normalized to $c_{0}$ will be given by $v_{p h}=\omega / k_{j 1}$ and the damping per unit wave length becomes $k_{j 1} / k_{j 2}$. In particular, from Eq. (2.6) follows that for $\theta=0$, $u_{f}^{2}=a^{2}$ and $u_{s}^{2}=c^{2}$, i.e., the fast and slow modes become an undamped Alfvén and a damped sound wave, respectively; and for $\theta=\pi / 2, u_{f}^{2}=a^{2}+c^{2}$ and $u_{s}^{2}=0$, i.e., only the fast magnetosonic damped wave propagates.

\section{Photoionized Hydrogen Plasma with Metallicity}

For context, the damping effect of the bulk viscosity on magnetosonic waves propagating in an optically thin hydrogen plasma with metallicity heated and ionized by a background radiation field of mean photon energy $E$ and ionization rate $\varsigma$ will be evaluated. For such a plasma the net rate function $X(\rho, T, \xi)$ and the net cooling rate per unit mass $L(\rho, T, \xi)$ are given, respectively, by

$$
X(\rho, T, \xi)=N_{0} \rho\left[\xi^{2} \alpha-(1-\xi) \xi \gamma_{c}\right]-(1-\xi)(1+\phi) \varsigma,
$$


$L(\rho, T, \xi)=N_{0}^{2} \rho\left[(1-\xi) Z \Lambda_{H Z}+\xi Z \Lambda_{e Z}+(1-\xi) \xi \Lambda_{e H}+\xi^{2} \Lambda_{e H^{+}}\right]-N_{0}(1-\xi) \varsigma\left[E_{h}+(1+\phi) \chi_{h}\right]$.

(Corbelli \& Ferrara 1995), where $\phi$ is the number of secondary electrons, $N_{0}$ is the Avogadro number, $E_{h}$ is the heat released per photoionization (Shull \& Van Steenberg 1985); $\Lambda_{H Z}, \Lambda_{e Z}, \Lambda_{e H}$ and $\Lambda_{e H^{+}}$are, respectively, the cooling efficiencies by collisions neutral hydrogen-ions and metal atoms (Launay \& Roueff 1977, Dalgarno \& McCray 1972), electrons-ions and metal atoms (Spitzer 1978), Ly $\alpha$ emission by neutral hydrogen Dalgarno \& McCray 1972) and hydrogen recombination, on the spot approximation (Seaton 1959).
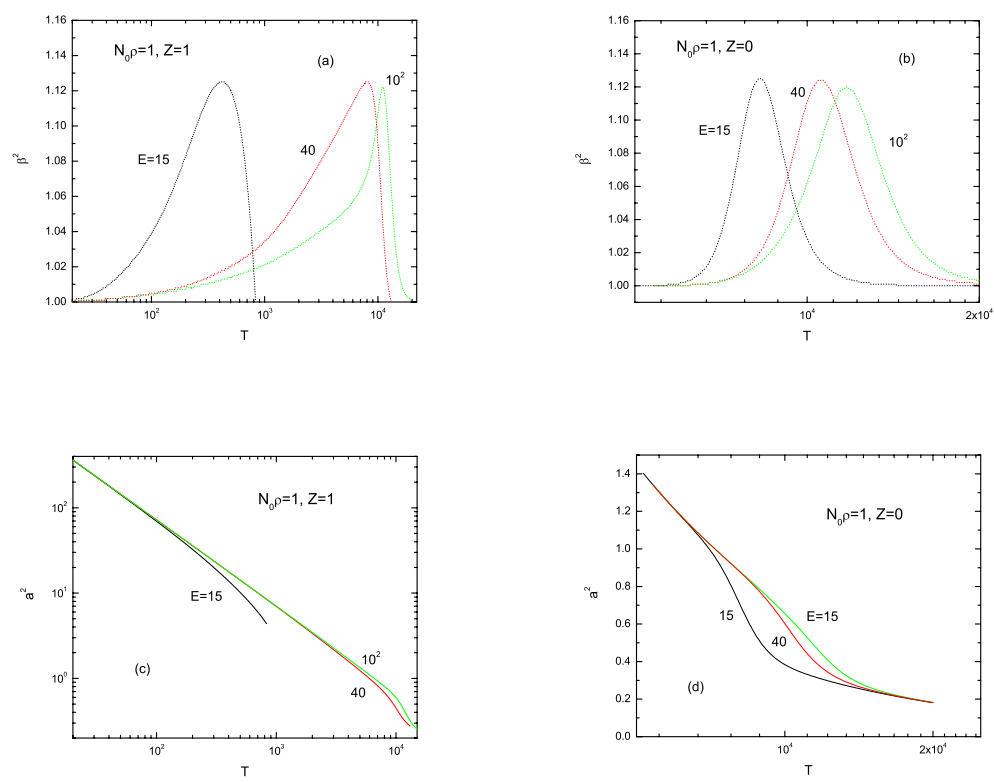

Figure 1. Two-layer model depicting a stratified solar atmosphere. The lower part of the atmosphere (index 1) is separated from the upper part (index 2) by a density and temperature discontinuity at $z=L$. Waves are launched at $z=0$ and propagate in the vertical $z$-direction.

Figures 1(a) and 1(b) are plots of the parameter $\beta^{2}$ as a function of temperature (in the rage where the equilibrium $X=0$ and $L=0$ exists) for a plasma with solar abundances $Z=1$ and a pure hydrogen gas $Z=0$, respectively. In both plots $N_{0} \rho=1$ and curves for three values of $E=15,40$ and $10^{2} \mathrm{eV}$ are shown. The ratio $c_{\infty}^{2} / c_{0}^{2}>1$, regardless of the value of the temperature and of the values of the free parameters $N_{0} \rho$ and $Z$. However, the temperature at which a maximum is reached strongly depends on the photon energy $E$, but its value depends weakly on $E$.

In Figs 1(c) and 1(d) the ratio $a^{2}$ between the Alfvén velocity $H^{2} / 4 \pi \rho$ and the sound velocity at the equilibrium $c_{0}^{2}$ (second relation in Eq. (3.3)) are plotted as a function of temperature for $H=2 \times 10^{-6} G$ (mean value of the galactic magnetic field), with the values of the remaining free parameters corresponding to Figs 1(a) and 1(b), respectively. For a plasma with solar abundances, $a^{2}$ strongly decreases with temperature (see Fig. 1(c)) due to the strong increase of the equilibrium sound speed $c_{0}^{2}$ when $T$ in- 
creases. Instead, for a pure hydrogen plasma such decrease is weak as it is apparent from Fig. 1(d), i.e., for a given value of the magnetic field the above ratio is very sensitive to the metallicity of the plasma and weakly depends on the photoionizing energy $E$.
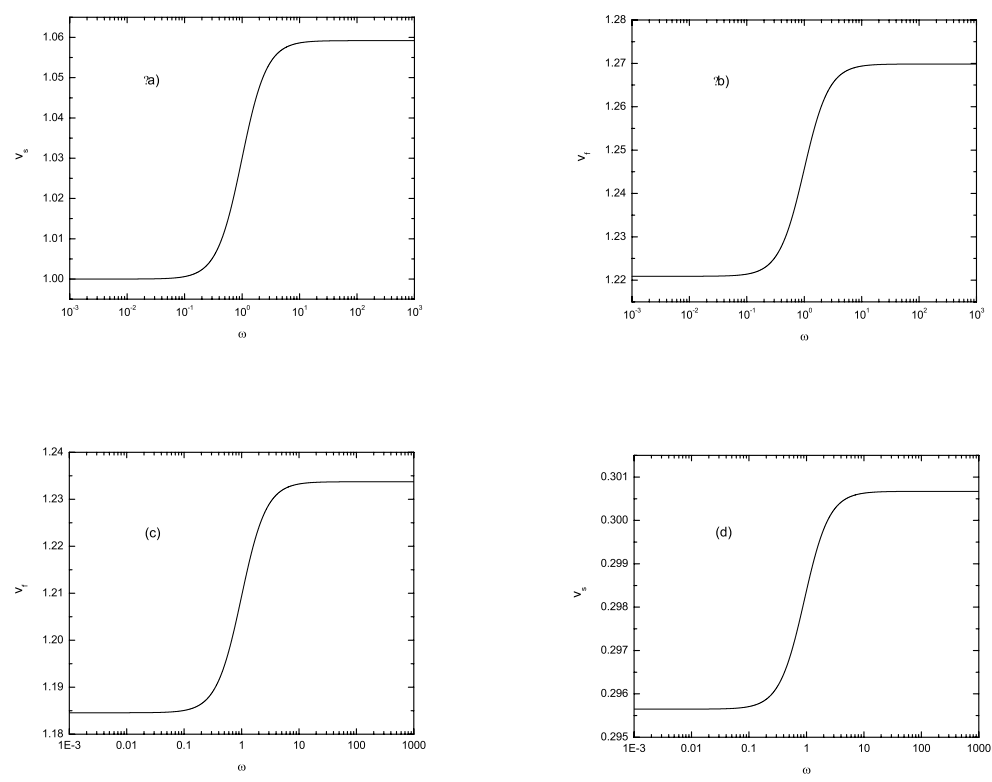

Figure 2. The phase velocity of the sonic (a) and magnetosonic (b) mode for $N_{\circ} \rho=1, Z=1$, $E=10^{2} \mathrm{eV}$ and $\theta=0$ (a) and $\theta=\pi / 2$.(b), $\theta=\pi / 3$ (c) and (d)

Figures 2(a) and 2(b) are plots of the phase velocity of both the sonic (slow mode) and the magnetosonic mode, as a function of dimensionless frequency for $\theta=0$ and $\pi / 2$, respectively, and a temperature $T=1.1 \times 10^{4} K$ at which $\beta^{2}$ is a maximum and for $Z=1, N_{0} \rho=1, E=10^{2} \mathrm{eV}$. The slow mode becomes a damped sonic mode which for $\omega<<1$ propagates with velocity $c_{0}$, as it apparent from Fig. 2(a). The fast mode becomes an Alfvén mode. For $\theta=\pi / 2$, the fast mode becomes a magnetosonic damped mode with phase velocity $u_{f}^{2}=a^{2}+c^{2}$ as shown in Fig. 2(b). The phase velocity becomes $v_{s}=0$ for the slow mode.

Figures 2(c) and 2(d) are plots of the phase velocity as function of the dimensionless frequency $\omega$ of the slow and fast modes, respectively, for $\theta=\pi / 3$. The respective damping per unit wave length $k_{j 1} / k_{j 2}(j=f, s)$ as a function of the dimensionless frequency $\omega$ is plotted in Figs. 3(a) for the slow mode when $\theta=0$ (solid line), the fast magnetosonic mode when $\theta=\pi / 2$ (dashed line), and for the fast and slow magnetosonic modes when $\theta=\pi / 3$ (dotted lines). The slow mode is less damped than the fast one. In all the above cases under consideration, the maximum damping occurs for values of the dimensionless frequencies close to 1 , i.e., where the period of the oscillation is close to the relaxation time $\tau$, and where the strongest change in the corresponding phase velocity also takes place (see Figs. 2 and 3).

In general, the same qualitative behavior is shown by the phase velocity and the respective damping for both, the slow and fast mode, when the parameters $Z, E, T$ and 

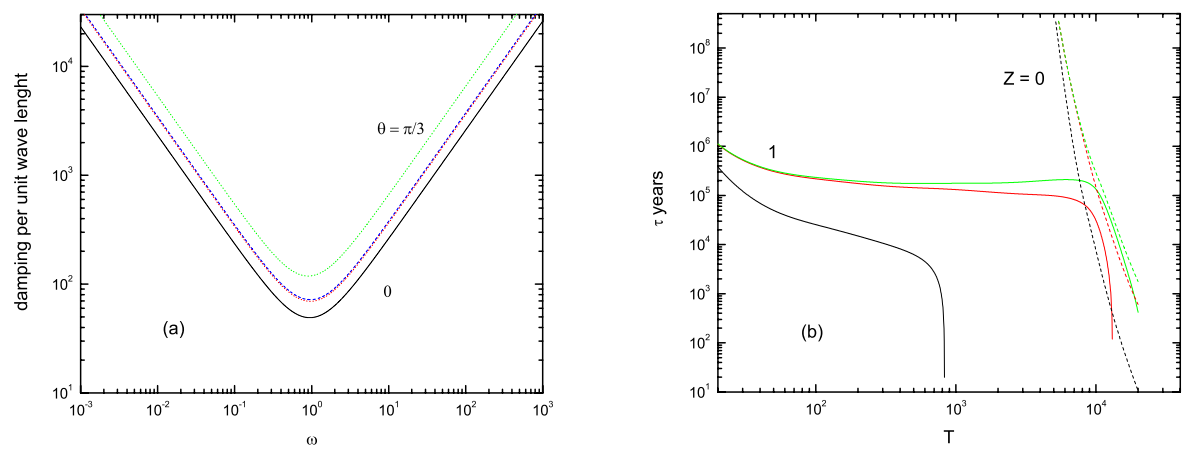

Figure 3. (a) The damping rate per unit of wave length as a function of the dimensionless frequency $\omega$ for the corresponding modes on Figs. 1(c) to 2(d).(b) The relaxation time $\tau$ (in years) as a function of temperature for plasmas with $Z=0$ (dashed lines) and $Z=1$ and $E=15,40$ and $10^{2} \mathrm{eV}$.
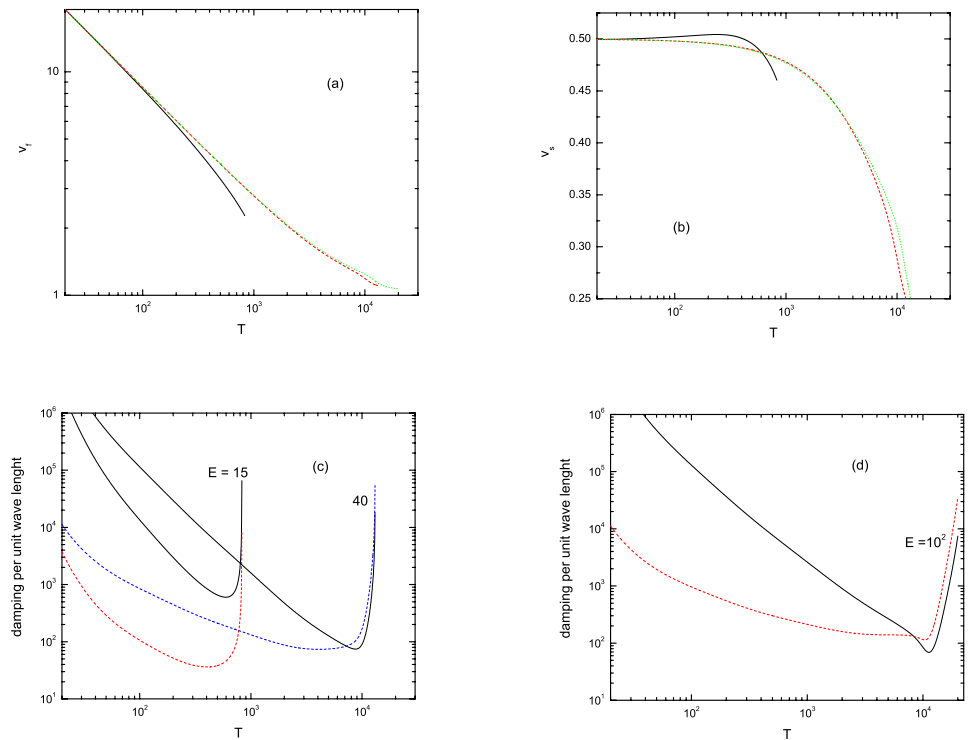

Figure 4. The phase velocity of the fast (a) and slow(b) mode as a function of temperature for a wave with maximum damping per unit wave length $(\omega=1)$, for $Z=1$ and three values of the photoionizing energy $E=15$ (solid lines), 40 (dash lines) and $10^{2} \mathrm{eV}$ (point lines). and the corresponding damping per unit wave length (c) and (d). Solid and dash lines correspond to the fast and slow mode, respectively.

$\mathbf{H}$ are varied. However, the time scales involved can be very different, as it can be seen from Fig. 3(b) where the relaxation time $\tau$ has been plotted as a function of the temperature for a hydrogen plasma (dashed lines) and for a plasma with solar abundances (solid lines). The two sets of three lines correspond to a photon energy of $E=10^{2}, 40$ and $15 \mathrm{eV}$, from the top for the set with $Z=1$ and from the right to the left for the set with $z=0$, respectively. 
In Fig. 4 the phase velocity of the fast and slow mode as well as the respective damping length are plotted as function of temperature for a dimensionless frequency $\omega=1$ (where the maximum damping occurs), for a plasma with $Z=1$, an angle $\theta=\pi / 3$, and three values of the photoionizing energy. The phase velocity of the fast and slow modes are shown in Figs 4(a) and 4(b), respectively, for the three photoionizing energies $E=15$ (solid lines), 40 (dash lines) and $10^{2} \mathrm{eV}$ (point lines). The corresponding damping per unit wave length is shown in Figures 4(c) and 4(d). Both velocities strongly decay with temperature, as it is expected from simple physical arguments. The strongest damping occurs at higher plasma temperature where the energy transfer becomes much more effective. Finally, one can point out that the damping becomes weaker when the strength of the magnetic field increases.

\section{Summary}

In summary, in reacting plasmas, magnetosonic waves are damped by bulk viscosity; the strongest damping occurs at frequencies $\omega$ close to the inverse of the chemical relaxation time $(1 / \tau)$ at which the strongest change of the phase velocity for both fast and slow modes also occurs. Depending on the value of the angle between the wave number and the magnetic field mode, crossing may occur. The above effects are shown, in particular, for a photoionized (by photons with mean energy $E$ ) hydrogen plasma with arbitrary metallicity $Z$ and particle number density $N_{0} \rho$ embedded in a magnetic field of the order of the observed mean galactic magnetic field $(\sim 2 \mu G)$.

\section{Acknowledgements}

This work has been supported by CDCHT, Universidad de los Andes, Project C-149307-05-B

\section{References}

Braginskii, S. I. 1965, Rev. Plasma Phys. 1, 205

Carbonell, M. Oliver, R. and Ballester, J.L. 2004, A $\mathscr{E} A$ 415, 737

Corbelli, E. \& Ferrara, A. 1995, ApJ 447, 720

Dalgarno, A. \& McCray, R. A. 1972, ARAA 10, 375

Hennebelle, P \& Passot, T. 2006, A\&A 448, 1083

Heiles, C. \& Troland, T. 2005, ApJ 624, 773

Ibáñez, S. M. H. 2004, Phys. Plasmas 11, 5190

Ibáñez S.M. H \& Escalona, T.O. B. 1993, ApJ 415, 335

Kumar, N., Kumar, P. \& Singh, S. 2006, A\&A 453, 1067

Kumar, N. \& Kumar, P. 2006, Solar Phys 236, 137

Landau, L. D. \& Lifshitz, E.M. 1966 Electrodynamics of Continuous Media (Pergamon, London).

Landau, L. D.\& Lifshitz, E. M. 1987, Fluid Mechanics (Pergamon Press, London)

Launay, J. M \& Roueff, E. A\&A 1977, 56, 289

Moortel, De \& Hood, A. W., 2003 A\& A 408, 755.

Parker, E. N. 1979, Cosmical Magnetic Field (New York, Oxford University Press)

Roberts, B. 2000, Solar Phys. 193, 139

Seaton, M. J. MNRAS 1959, 119, 81

Shull, J. M. \& Van Steenberg, M. E. 1985,ApJ 298, 268

Spitzer, L. 1978, Physical Processes in the Interstellar Medium (New York: Wiley) 